Quantum coherence effects in atomic media such as electromagnetically-induced transparency and absorption, lasing without inversion, super-radiance and gain-assisted superluminality have become well-known in atomic physics. But these effects are not unique to atoms, nor are they uniquely quantum in nature, but rather are fundamental to systems of coherently coupled oscillators. In this talk I will review a variety of analogous photonic coherence phenomena that can occur in passive and active coupled optical resonators. Specifically, I will examine the evolution of the response that can occur upon the addition of a second resonator, to a single resonator that is side-coupled to a waveguide, as the coupling is increased, and discuss the conditions for slow and fast light propagation, coupled-resonator-induced transparency and absorption, lasing without gain, and gain-assisted superluminal pulse propagation. Finally, I will discuss the application of these systems to laser stabilization and gyroscopy. 


\title{
Coherence Phenomena in Coupled Optical Resonators
}

\author{
Dave Smith
}

NASA

Marshall Space Flight Center

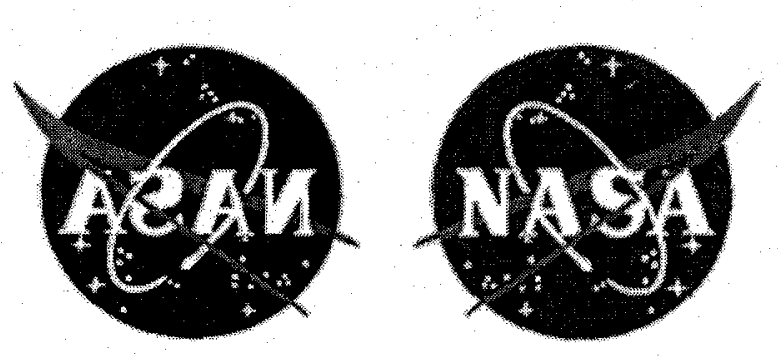




\section{Outline}

$>$ WGM Splitting

$>$ Coherence Effects in Passive Systems

- Coupled-Resonator-Induced Transparency and Absorption (CRIT and CRIA)

- Gain-Assisted Superluminality (GAS)

$>$ Coherence Effects in Active Systems

- Lasing Without Gain (LWG)

* Reduced lasing thresholds

$>$ Application: laser gyroscopy 


\section{- Varieties of Coupled Optical Resonators}

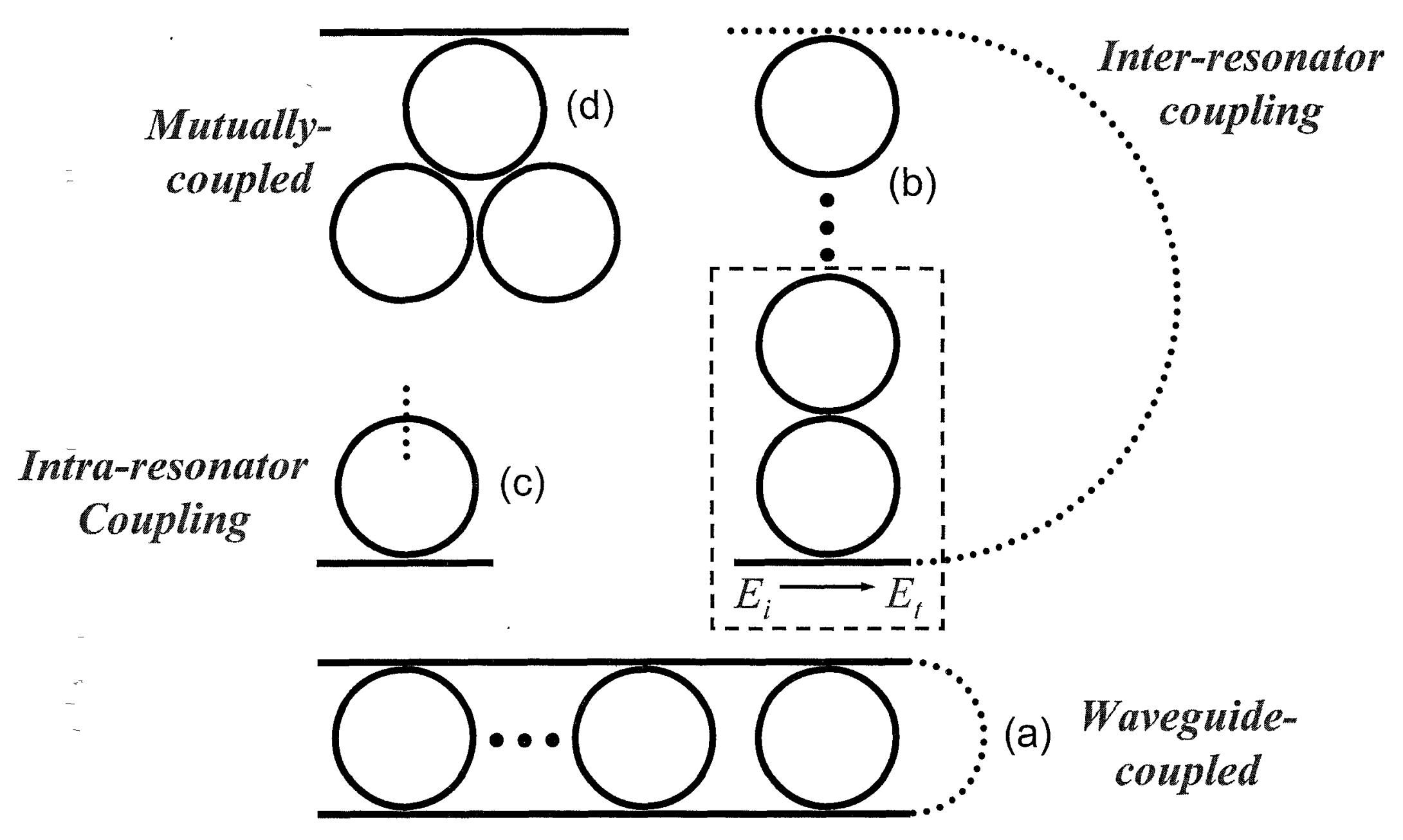




\section{Whispering-Gallery-Mode Splitting}

D.D. Smith, H. Chang, K. Fuller, JOSA B 20, 1967 (2003).

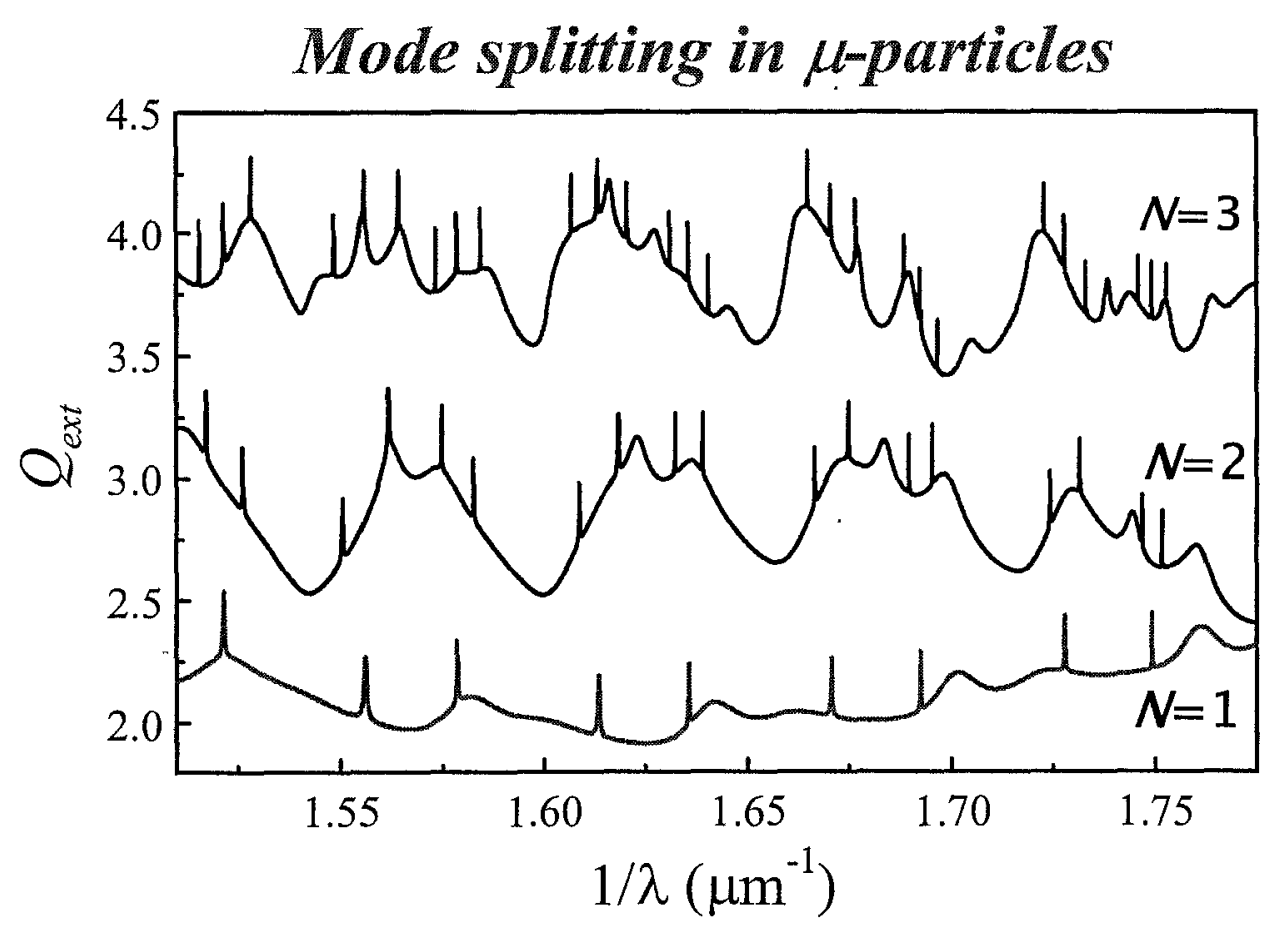

Mode splitting in ring resonators

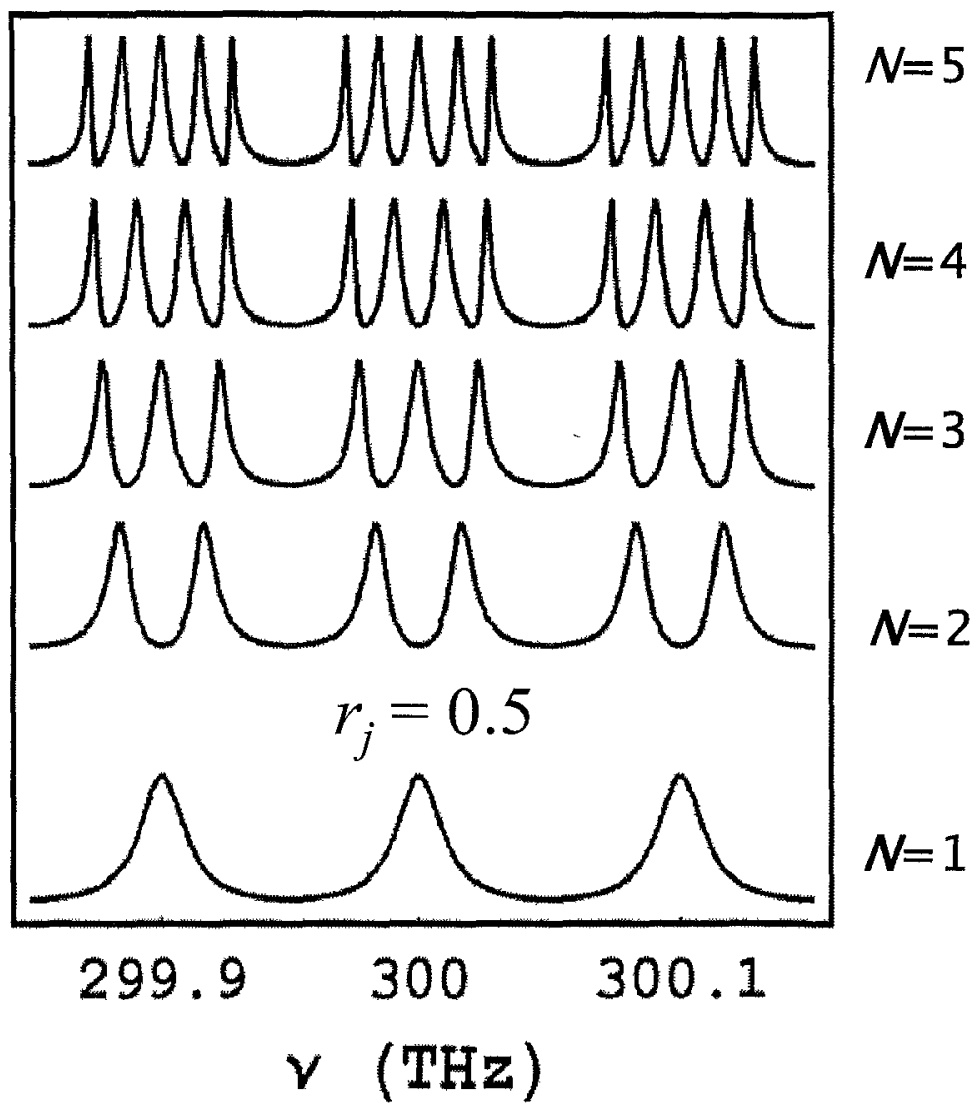

- Consequence of constructive ( $\mathrm{N}$ odd) or destructive ( $\mathrm{N}$ even) interference

- $N$ resonators yiel 


\section{- $\mid$ Tight-Binding PBGs in Photonic Molecules}
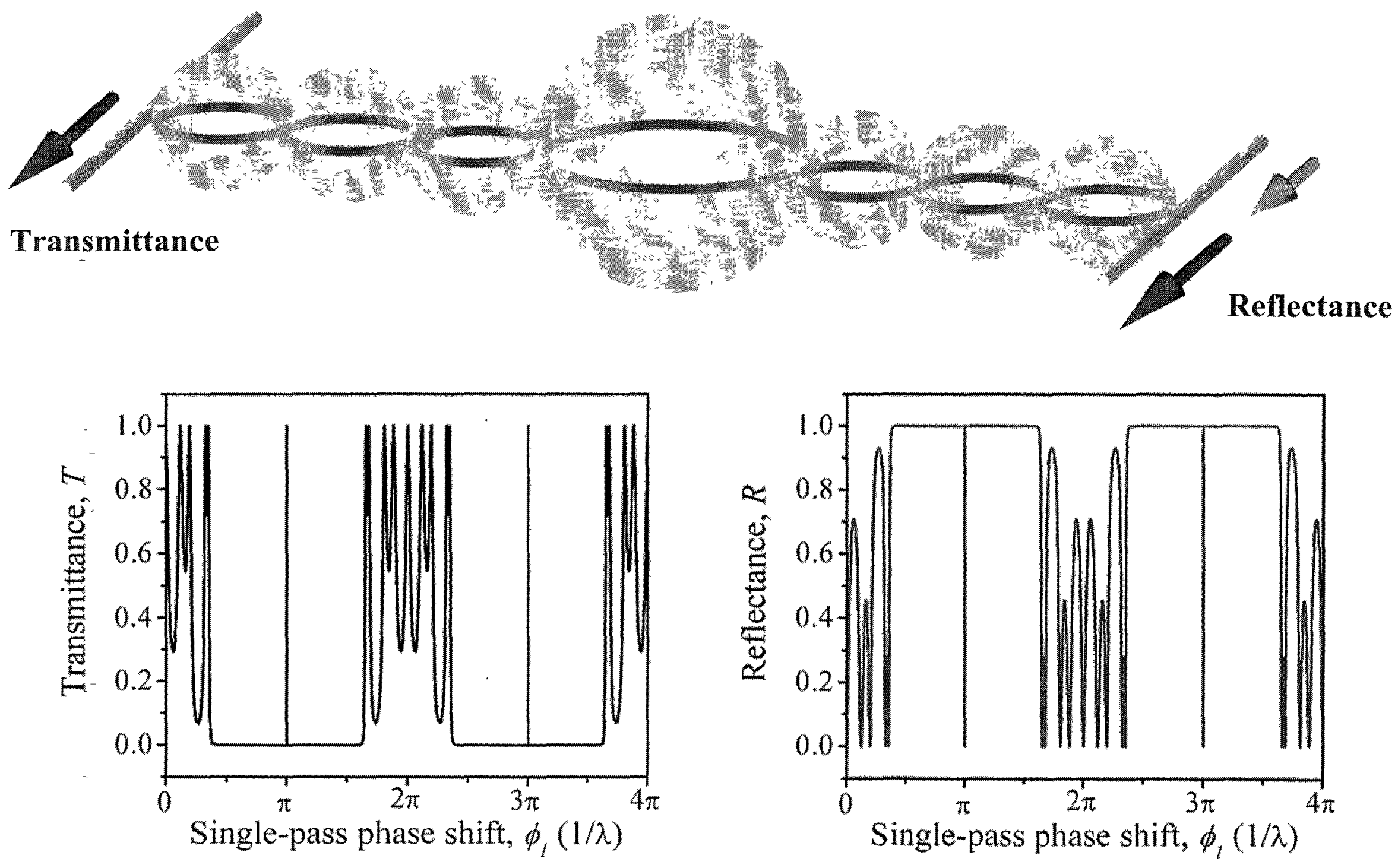


\section{Coupled Resonators $=$ Two Level Atom}

Coupled Modes = Schrod. Eqn. in RWA:

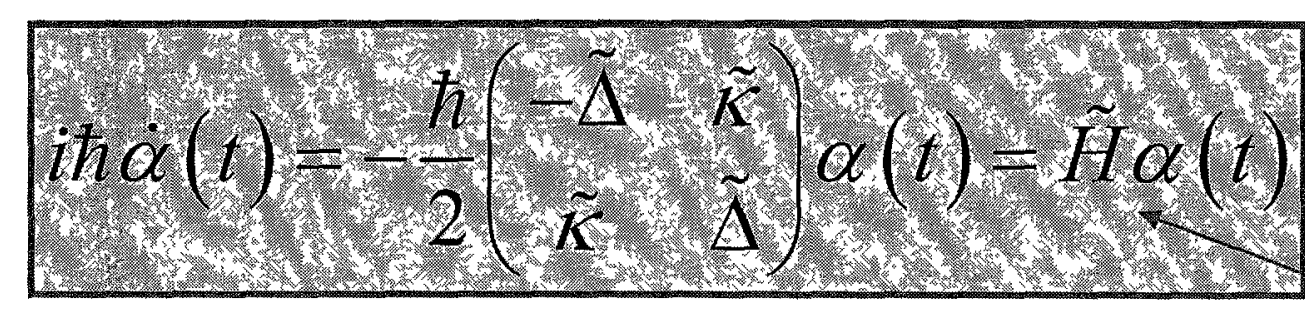

$\alpha(t)=\left[\begin{array}{ll}a_{1}(t) & a_{2}(t)\end{array}\right]^{\mathrm{T}}$ Rotating State

Coherent

Superposition

$11\rangle$

$$
|\psi(t)\rangle=E_{1}(t)|1\rangle+E_{2}(t)|2\rangle
$$

$\Omega_{R} \equiv \tilde{\kappa} \quad \tilde{\Delta} \equiv \tilde{\omega}_{1}-\tilde{\omega}_{2}=\Delta+i \gamma_{12} / 2 \quad$ Complex NL Coupling and Detuning

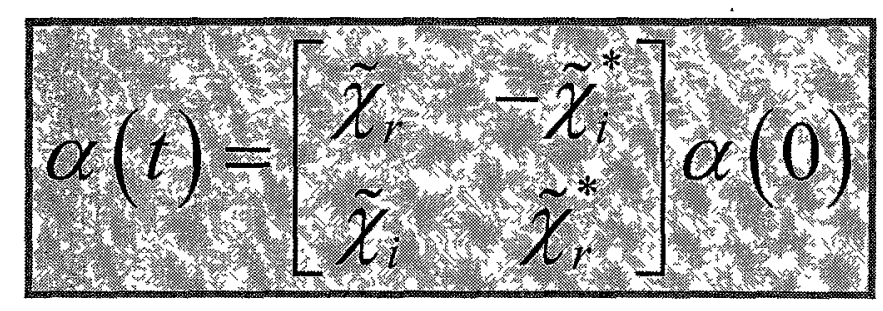

$\tilde{\Omega} \equiv \sqrt{\tilde{\Delta}^{2}+\left|\Omega_{R}\right|^{2}} \quad \begin{gathered}\text { Complex Generalized } \\ \text { Rabi Frequency }\end{gathered}$

$$
\begin{aligned}
& \tilde{\chi}_{r}=\cos (\tilde{\Omega} t / 2)+i(\tilde{\Delta} / \tilde{\Omega}) \sin (\tilde{\Omega} t / 2) \\
& \tilde{\chi}_{i}=i\left(\Omega_{R}^{*} / \tilde{\Omega}\right) \sin (\tilde{\Omega} t / 2)
\end{aligned}
$$

Non-Hermitian

Hamiltonian
Slow-varying amplitudes
Damped Rabi Oscillations?

If $\gamma_{12} \neq 0 \rightarrow \mathrm{H}$ is non-Hermitian, dressed states couple 


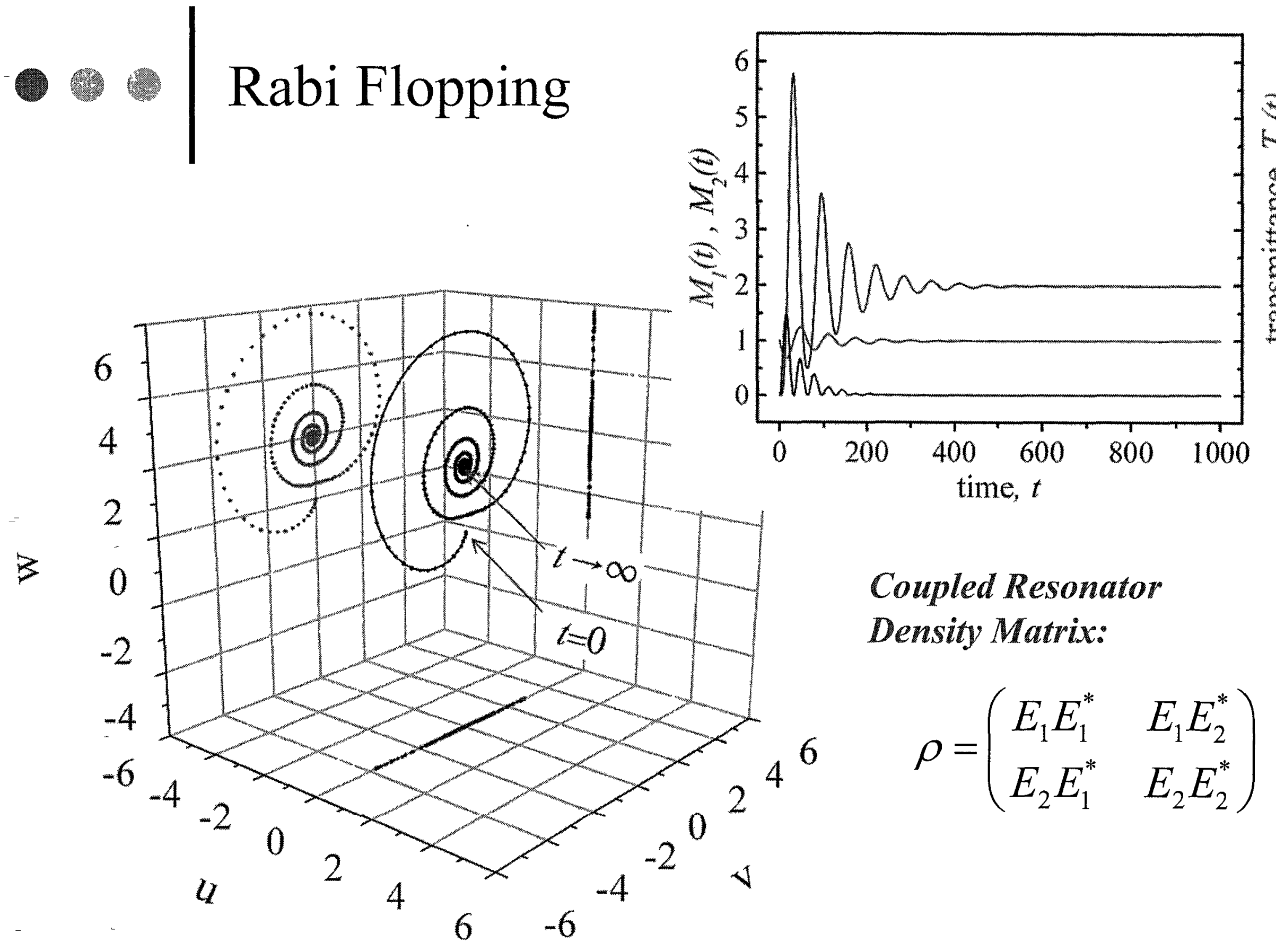



Coupled Resonators
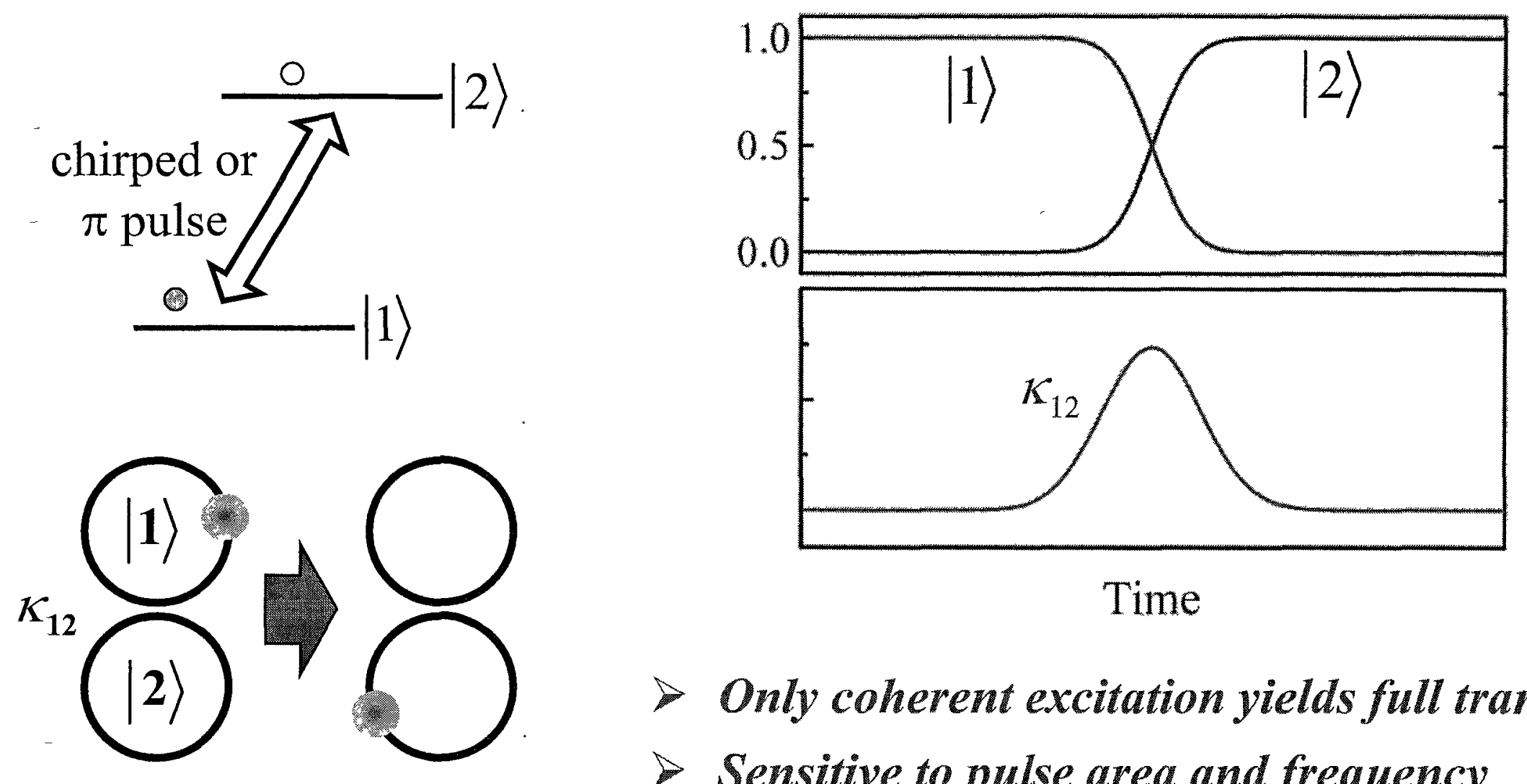

$>$ Only coherent excitation yields full transfer

$>$ Sensitive to pulse area and frequency

$>$ Adiabatic transfer independent of pulse area but requires long slow chirp 
Stimulated Raman Adiabatic Passage (STIRAP)
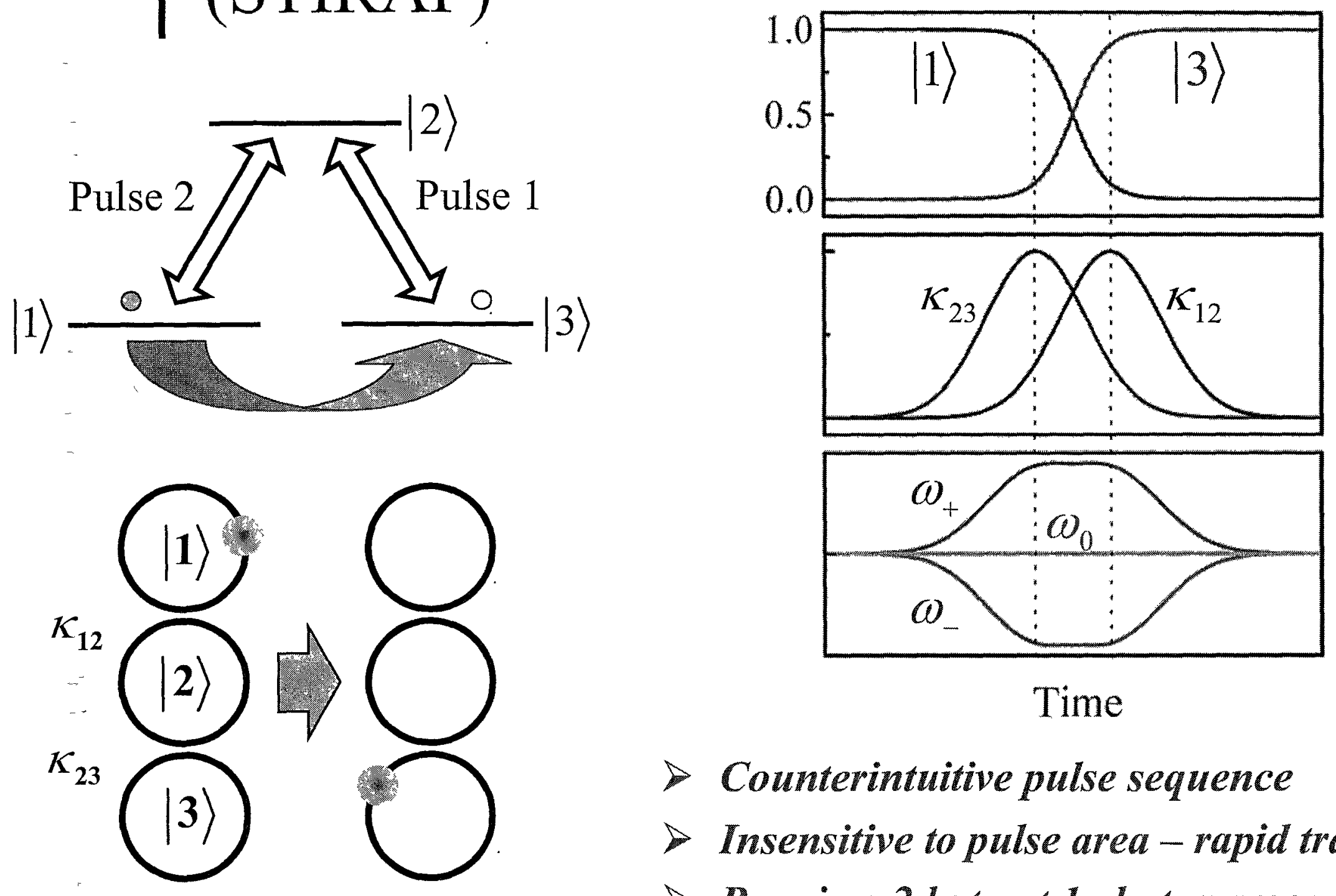

Time

$>$ Counterintuitive pulse sequence

- Insensitive to pulse area-rapid transfer

$>$ Requires 2 but not 1 photon resonance 


\section{Coupled-Resonator-Induced}

Transparency (CRIT)

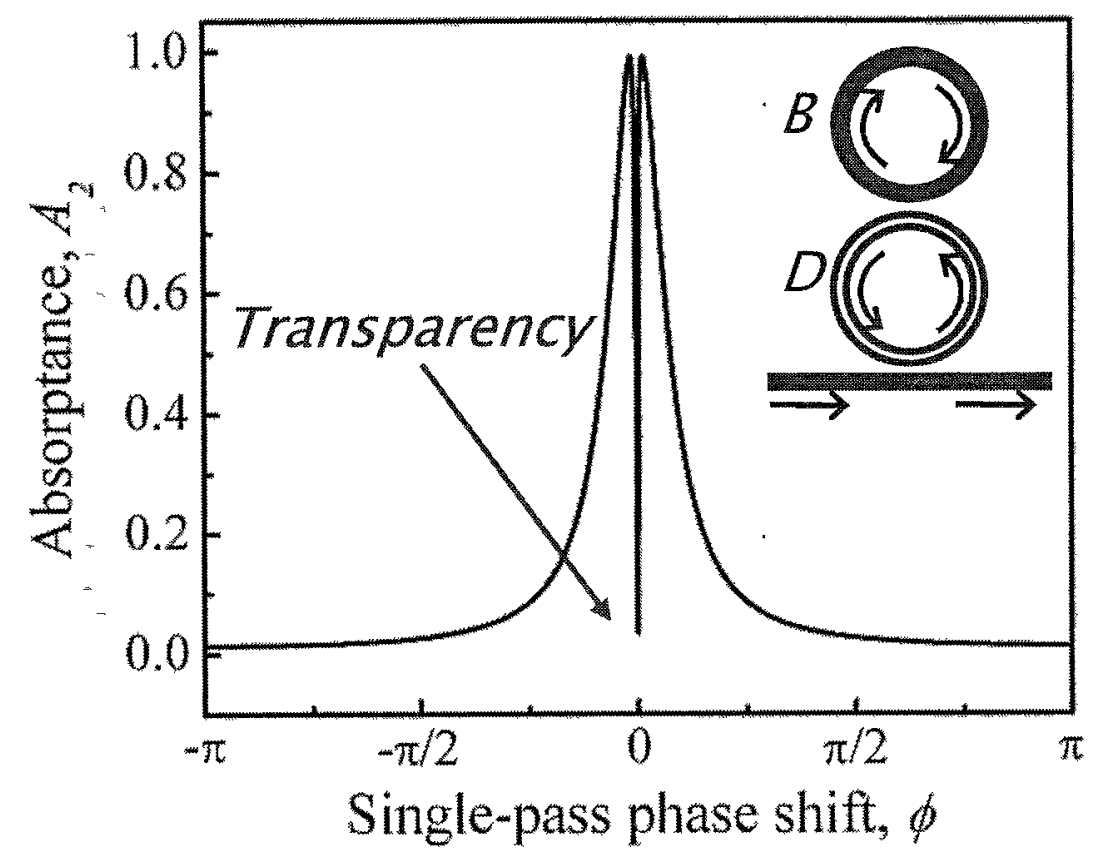

- Photon Trapping!

- Slow light with no absorption!

D.D. Smith, H. Chang, K. Fuller, A.T. Rosenberger, R.W. Boyd, PRA (2003).
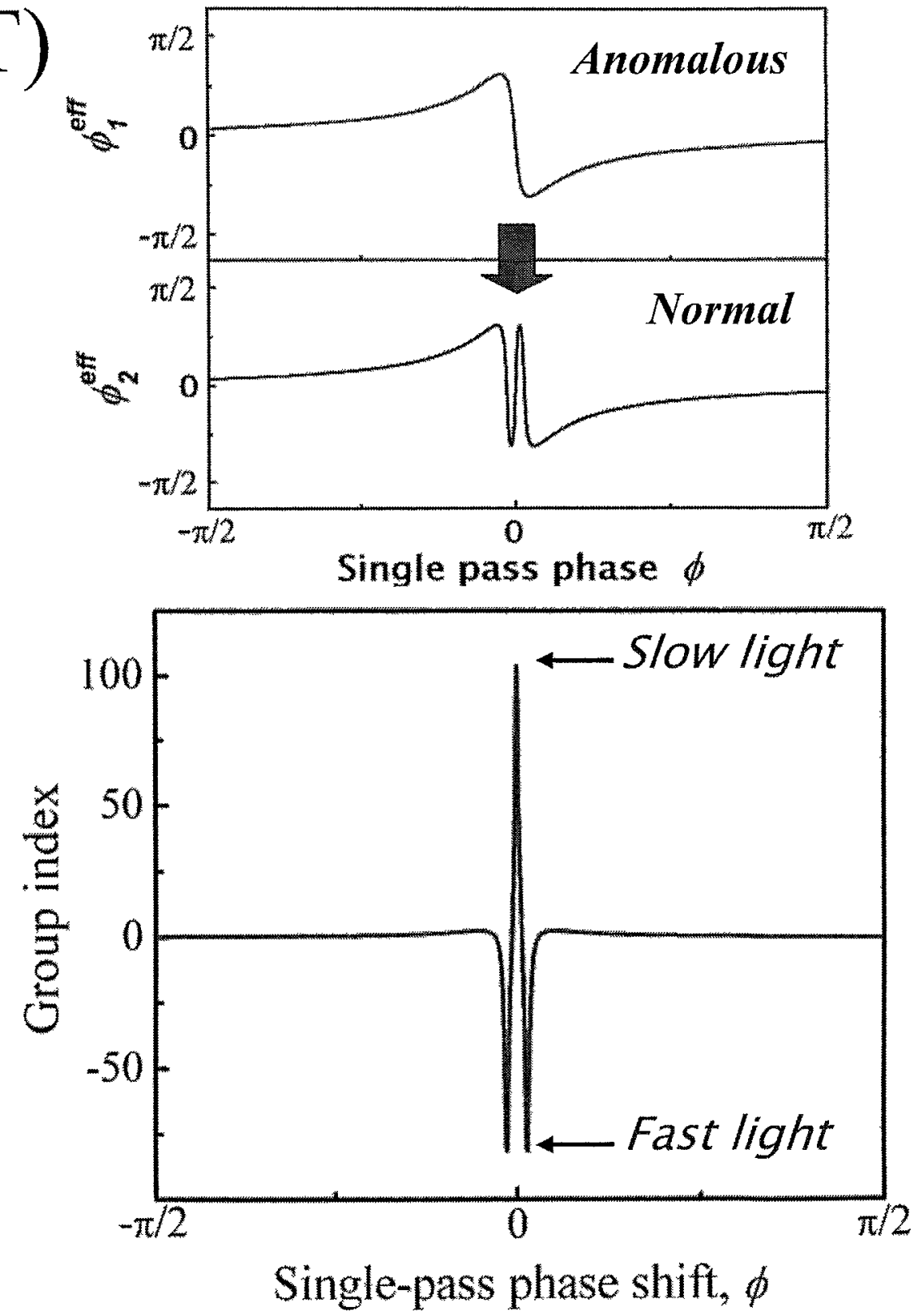


\section{Coupled-Resonator-Induced Absorption (CRIA)}

$>$ Analogous to Electromagnetically-Induced Absorption (EIA)

Typically results from Constructive Interference!

$>$ Requires the second resonator to be over-coupled $\left(r_{2}<a_{2}\right)$
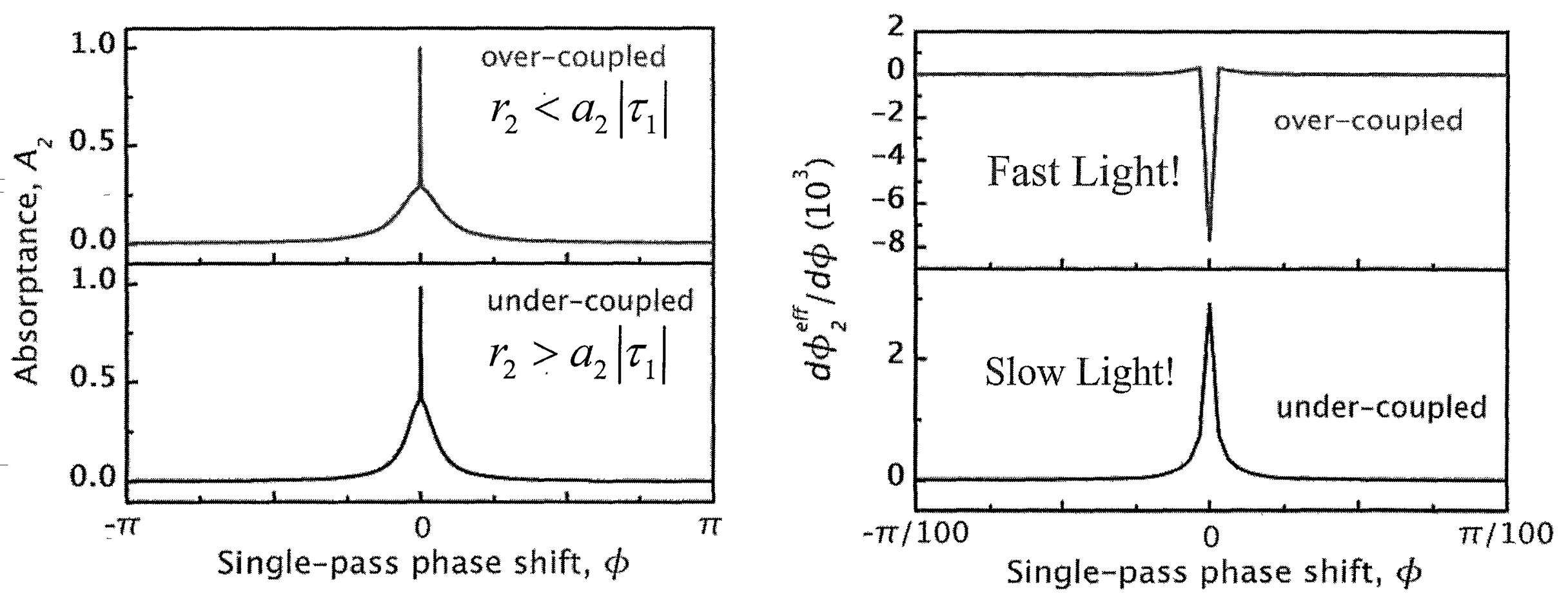


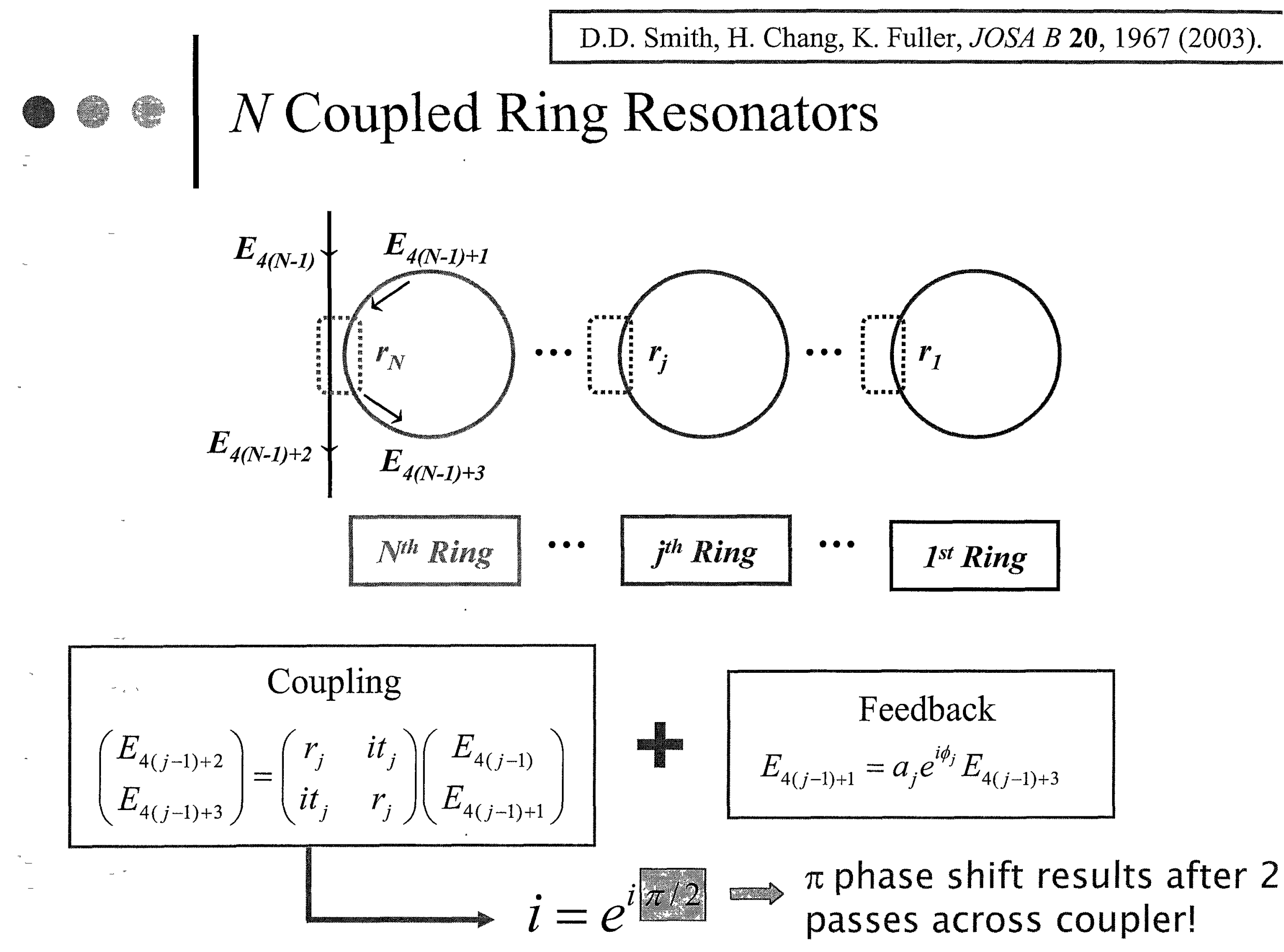




$$
\tau_{1}\left(\phi_{1}\right)=\frac{r_{1}-a_{1} e^{i \phi_{1}}}{1-r_{1} a_{1} e^{i \phi_{1}}}=\left|\tau_{1}\right| \exp \left[i \phi_{1}^{(\mathrm{eff})}\right] \quad \begin{gathered}
\text { Airy } \\
\text { Expressions }
\end{gathered}
$$

$\tau_{2}\left(\phi_{1}, \phi_{2}\right)=\frac{r_{2}-a_{2} \tau_{1} e^{i \phi_{2}}}{1-r_{2} a_{2} \tau_{1} e^{i \phi_{2}}}=\left|\tau_{2}\right| \exp \left[i \phi_{2}^{(\text {eff })}\right]$

$$
=\exp \left[i \tilde{\phi}_{2}^{\text {(eff) }}\right] \quad \tilde{\phi}_{2}^{\text {(eff) }}=\phi_{2}^{\text {(eff) }}-i \ln \left|\tau_{2}\right| \quad \text { Complex Phase }
$$

Dispersive Response

$$
\phi_{2}^{(\text {eff })}\left(\phi_{1}, \phi_{2}\right)=\arg \left(\tau_{2}\right)
$$

$>$ Slow or Fast Light

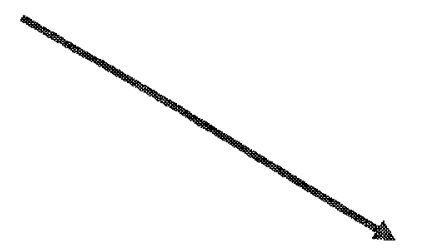

Absorptive Response

$$
T_{2}=\left|\tau_{2}\right|^{2} \quad A_{2}=1-T_{2}
$$

\section{CRIT or CRIA}




\section{Transmission Argand Diagrams}

\section{Single Resonator}

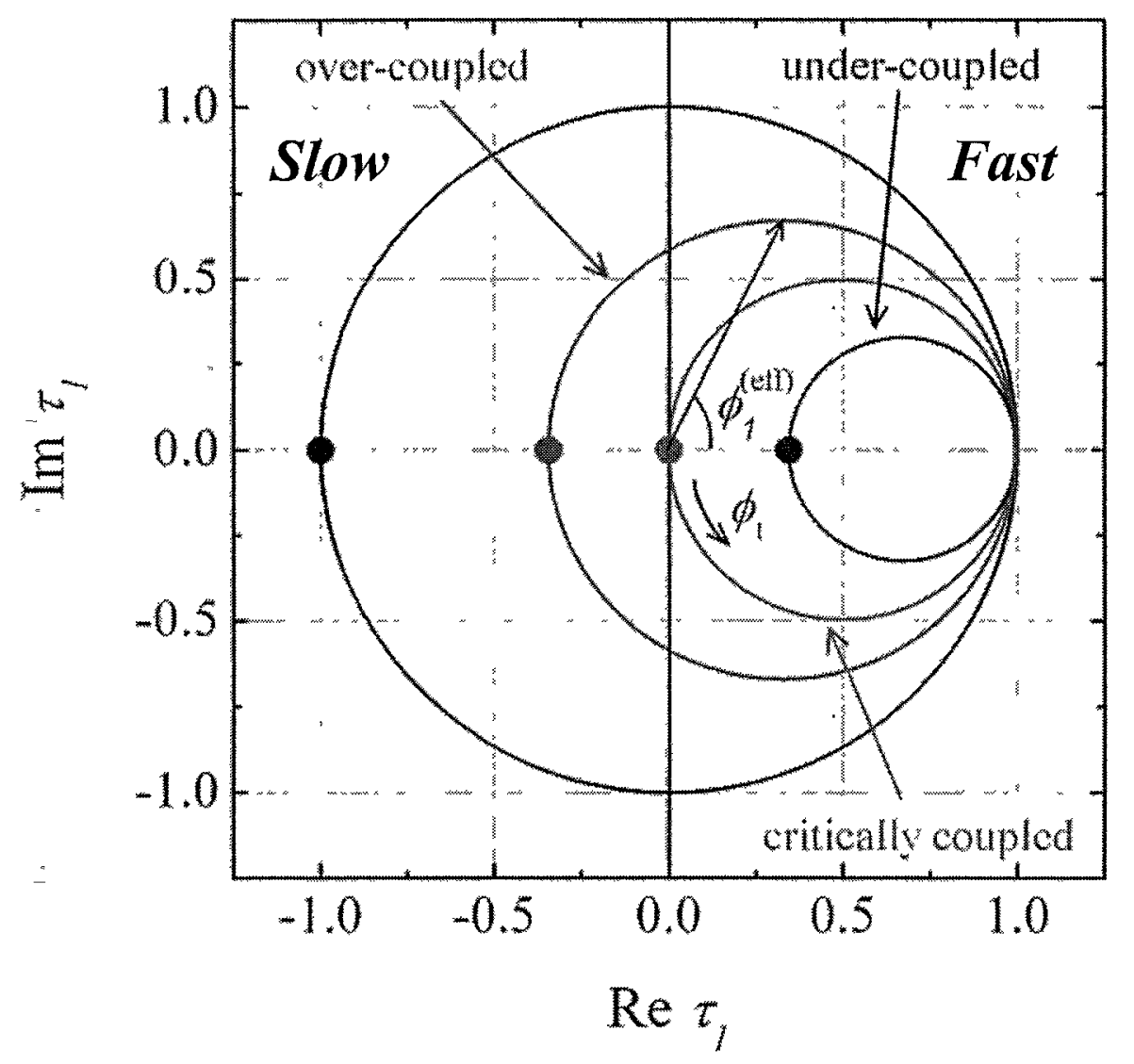

$>$ Slow light on the left!

\section{Two Resonators}

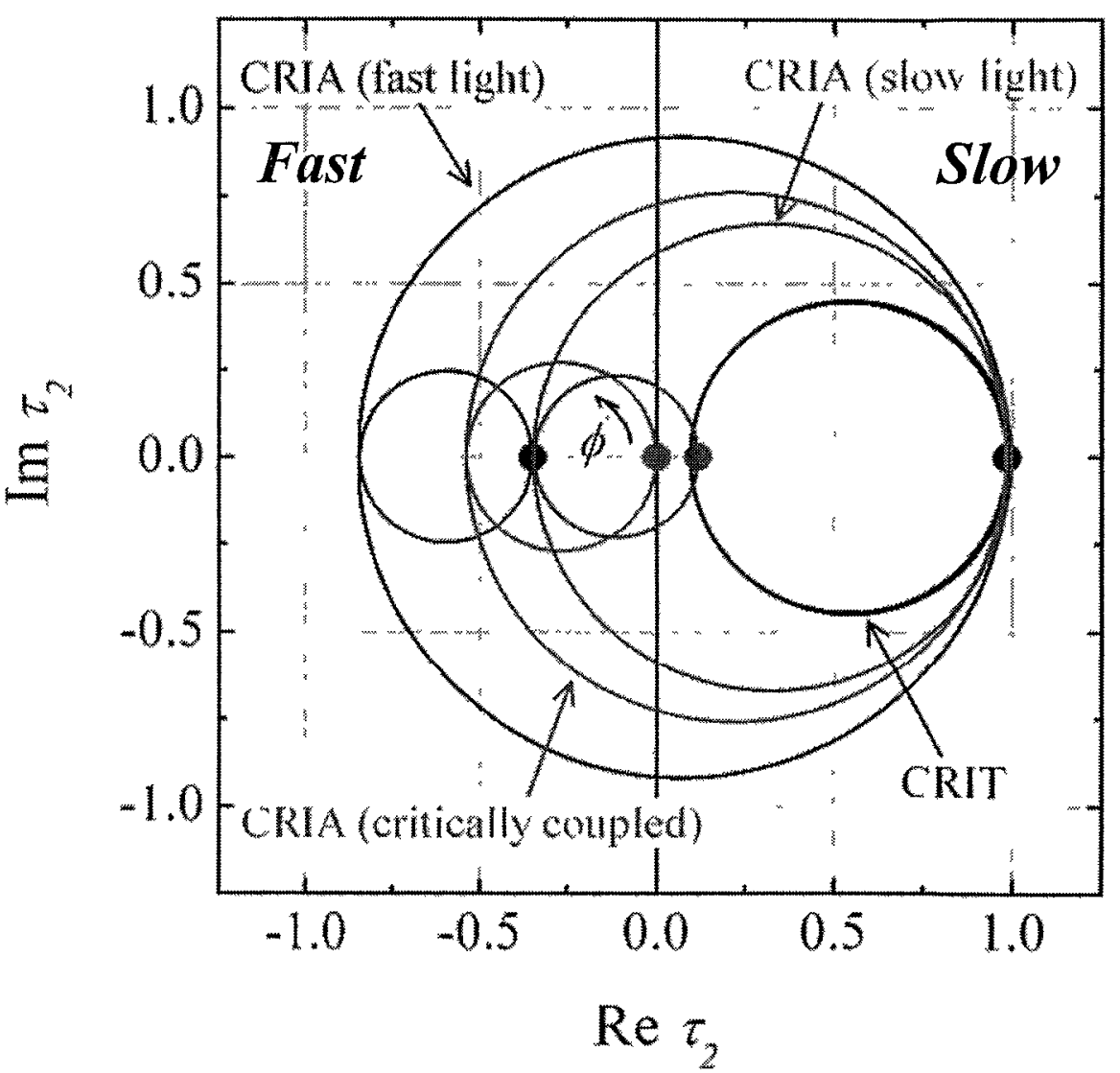

$>$ Slow light on the right! 


\section{Impulse Response}

\section{Coupled Resonators:}

Single Resonator:
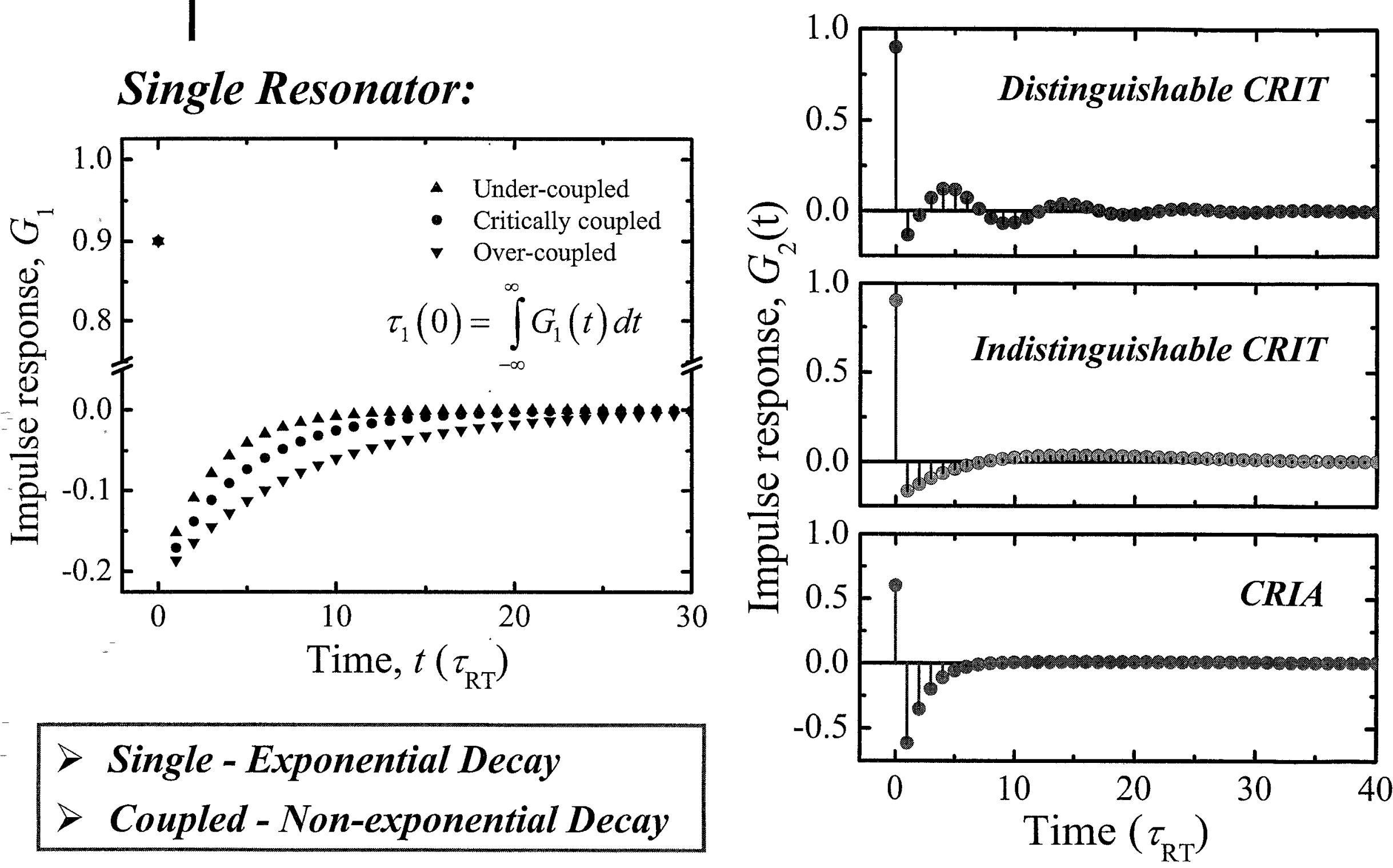
$\bullet \quad$ FDTD of CRIA and CRIT 
- $\mid$ Critical Coupling of CRIT and CRIA

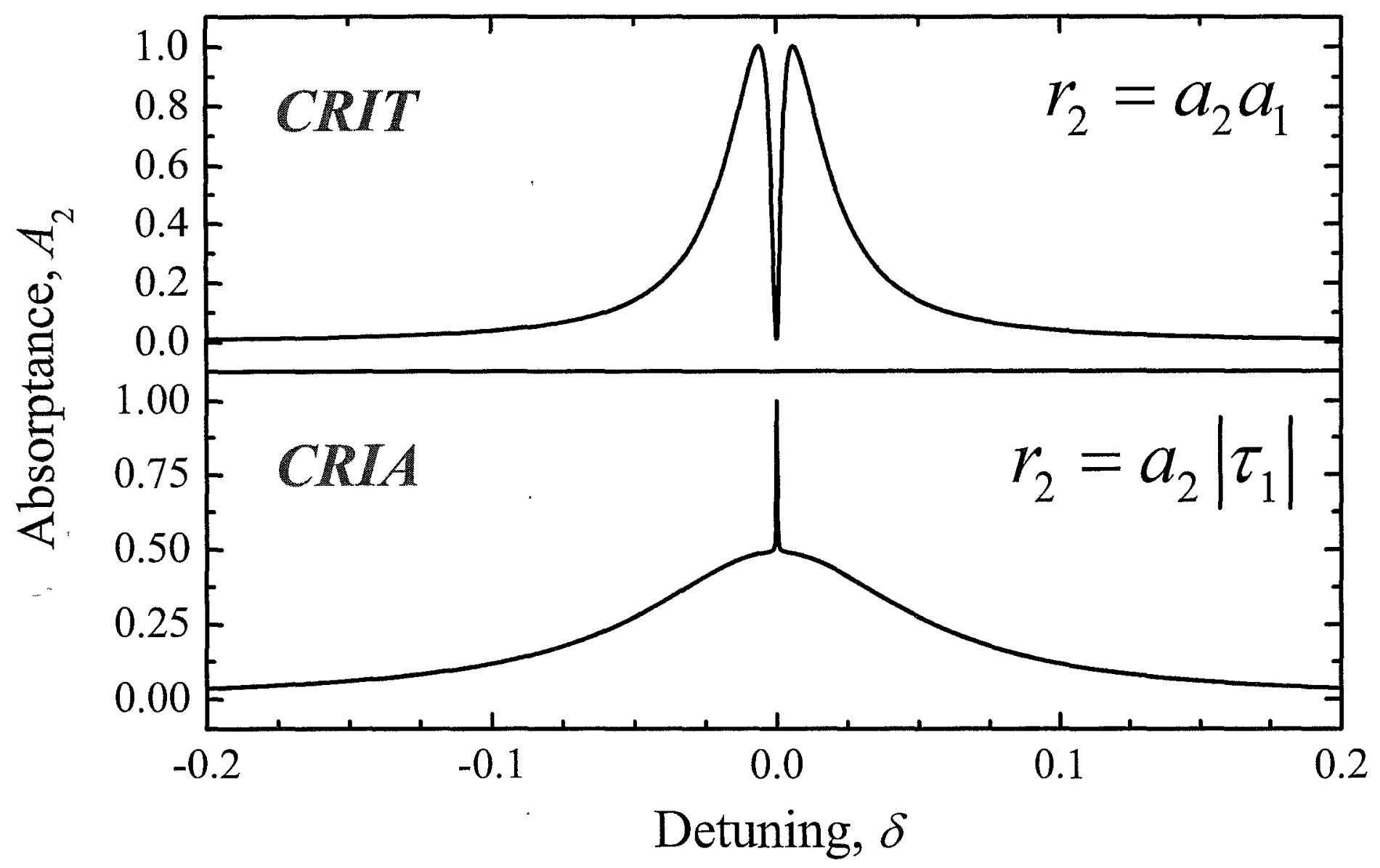




\section{Slow and Fast Pulse Propagation}
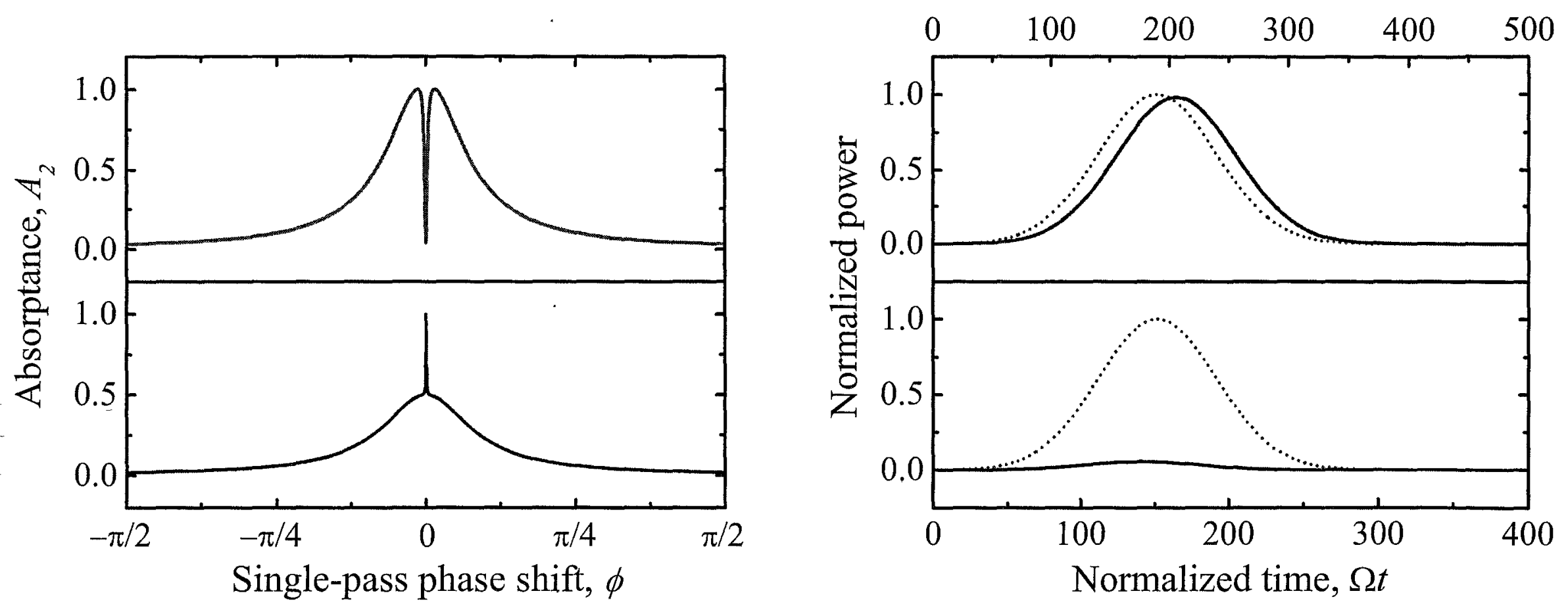

$>$ Slow light with no absorption

$>$ But fast light requires loss to obey causality / Relativity 
Gain-Assisted Superluminality

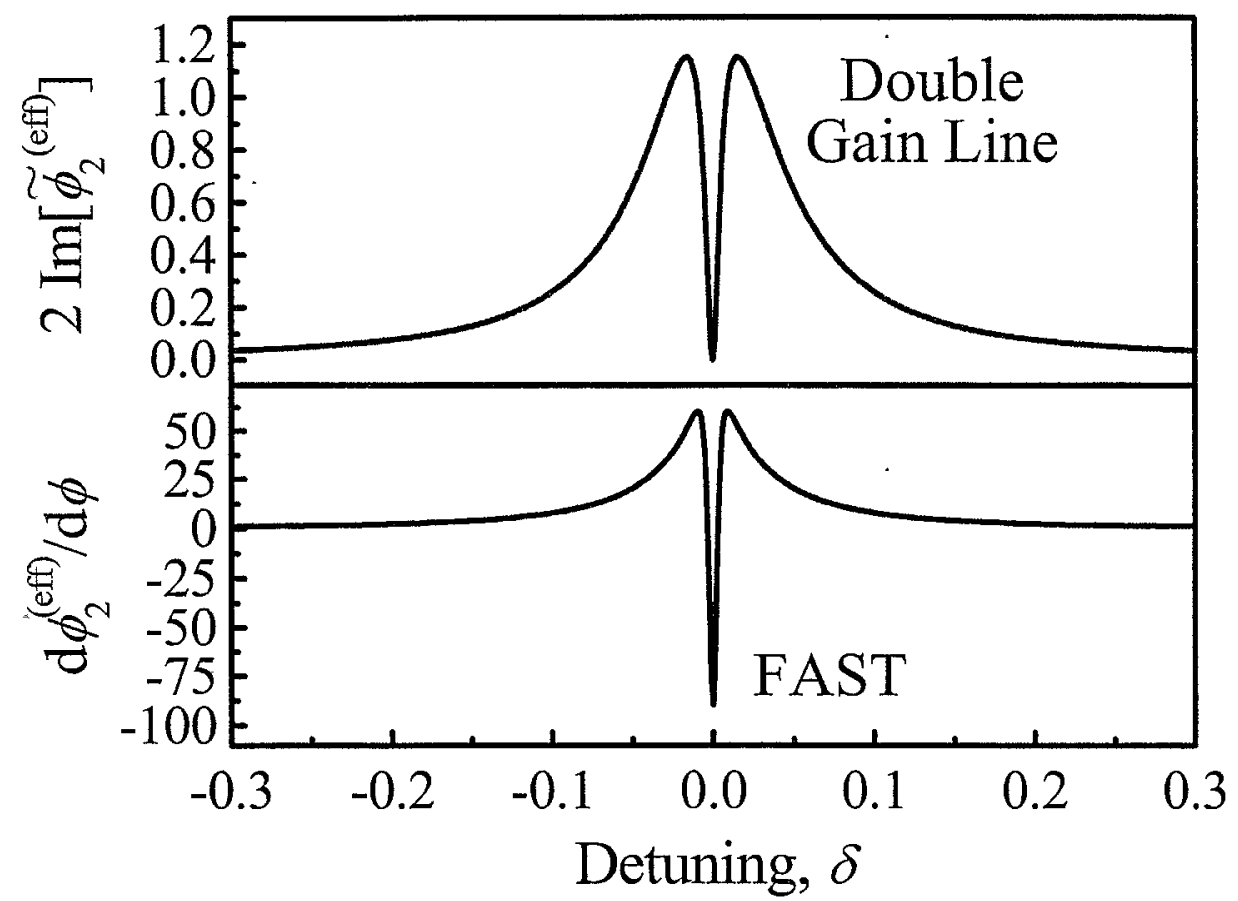

$>$ Fast light in a transparent medium!

$>$ Gain-assist reverses dispersion / CRIA boosted to transparency

$>$ Still does not violate causality

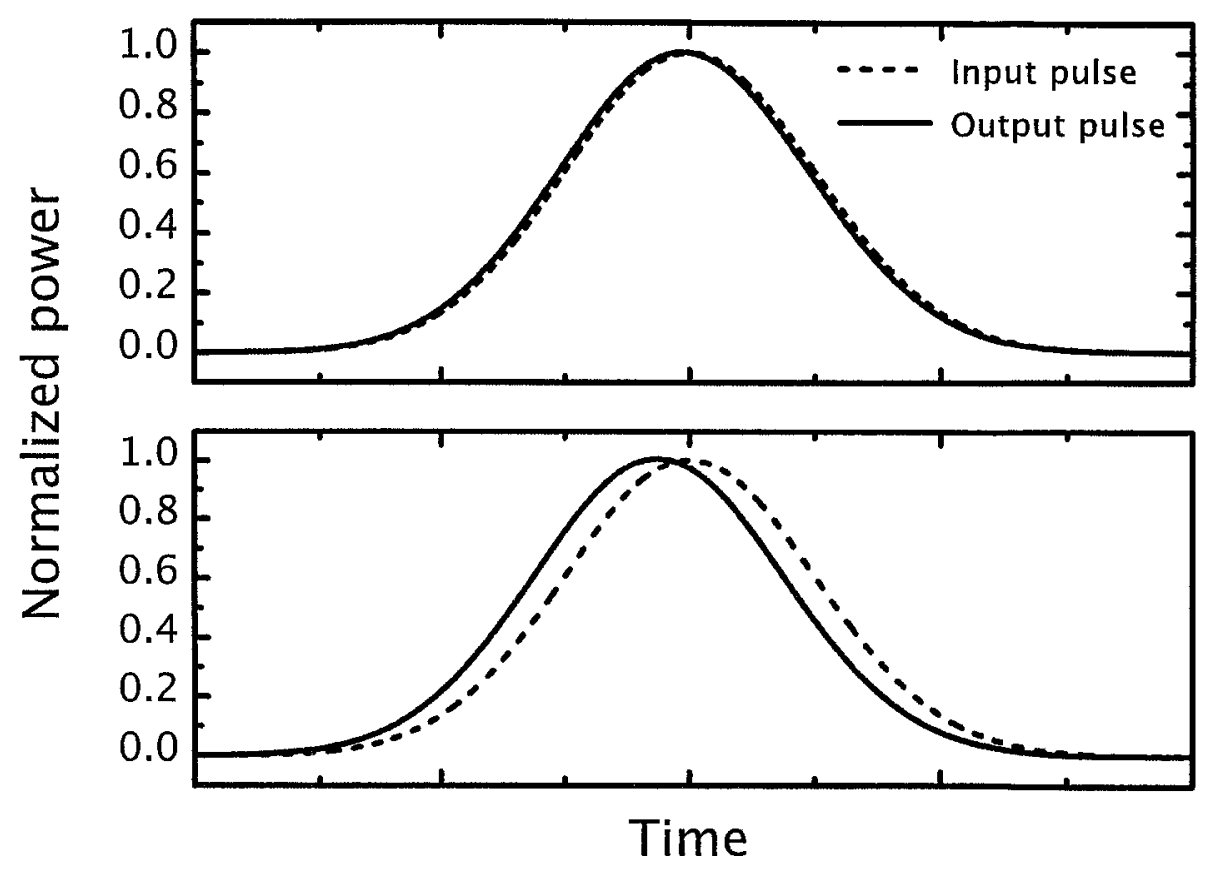

H. Chang, D.D. Smith, JOSA B (2005). 


\section{Lasing in Coupled Resonators}

$>$ Coherence affects lasing threshold!

$>$ LWI and Super-radiance analogs

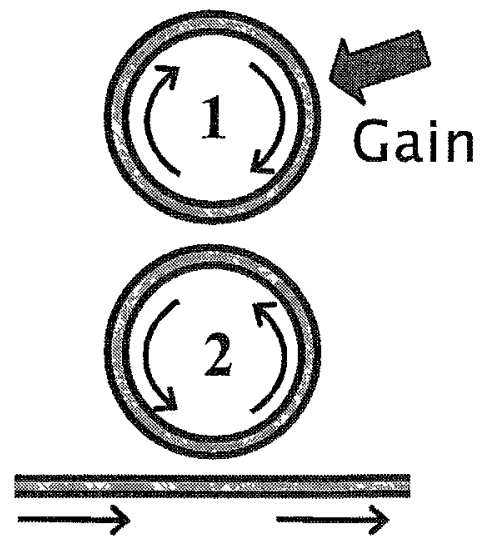

Distinguishable

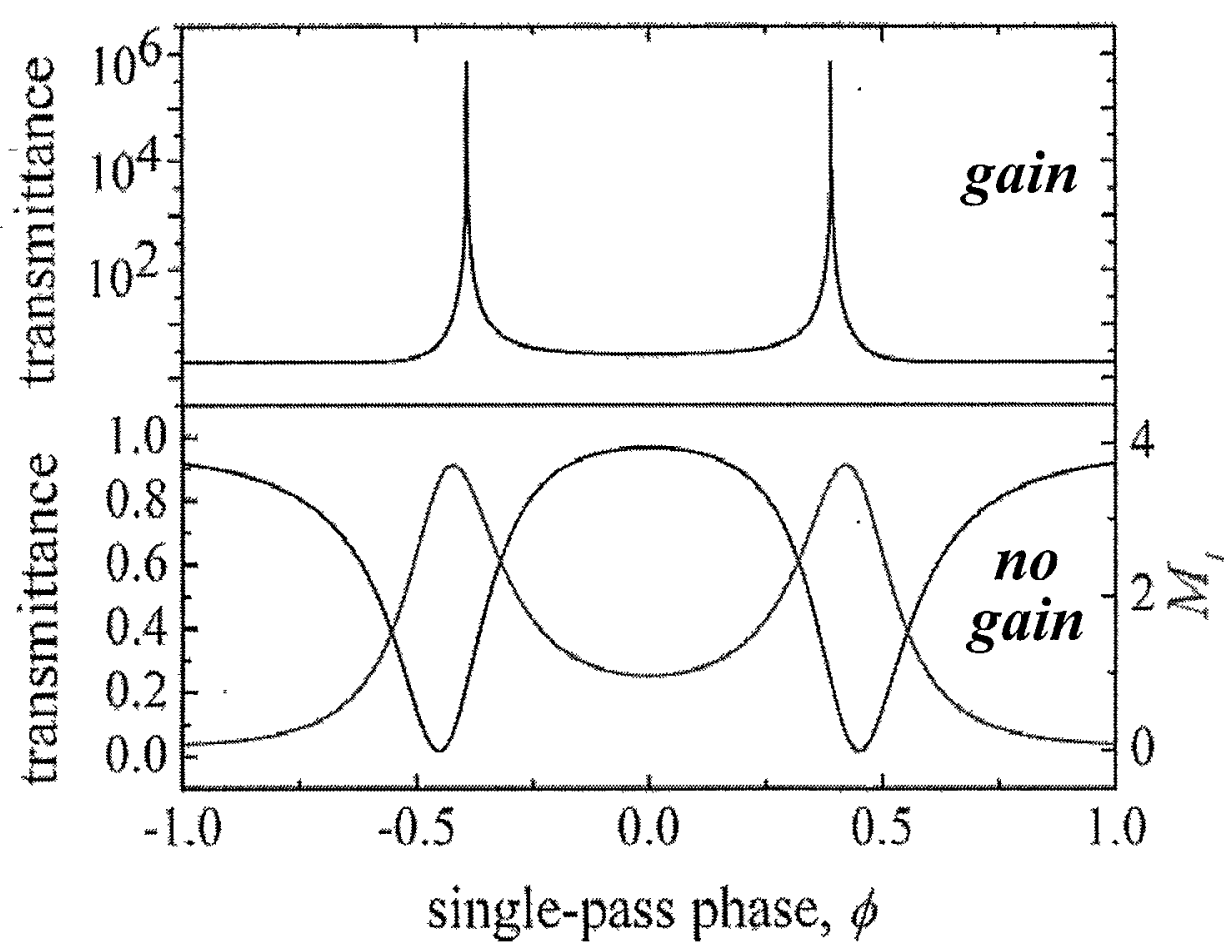

Indistinguishable

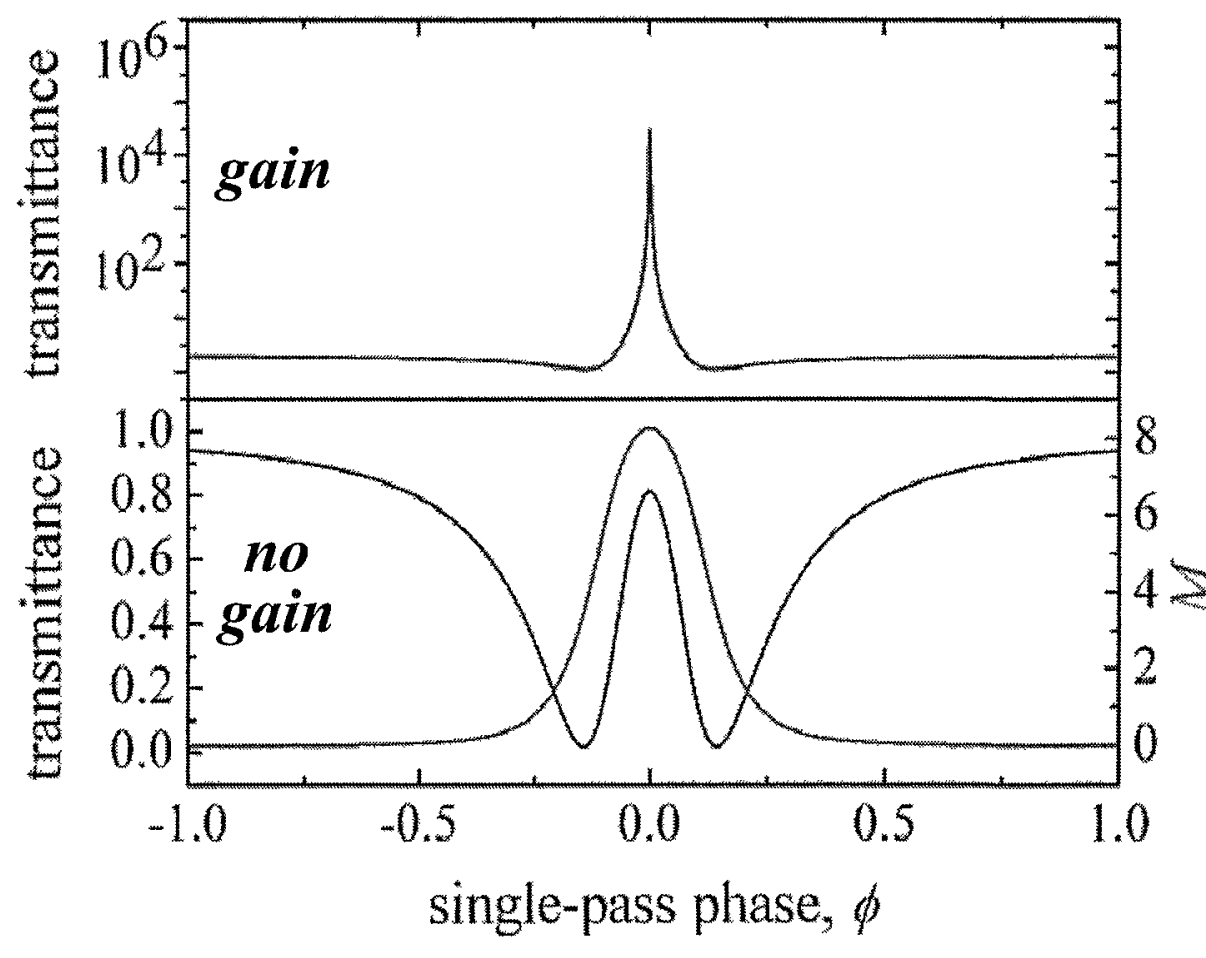




\section{Lasing Without Gain in Coupled Resonators}

- Analogous to LWI $\left(N_{e}<N_{g}\right)$ : Gain in resonator $1<$ Loss in resonator 2

- Lasing occurs by photon trapping - indistinguishability required.

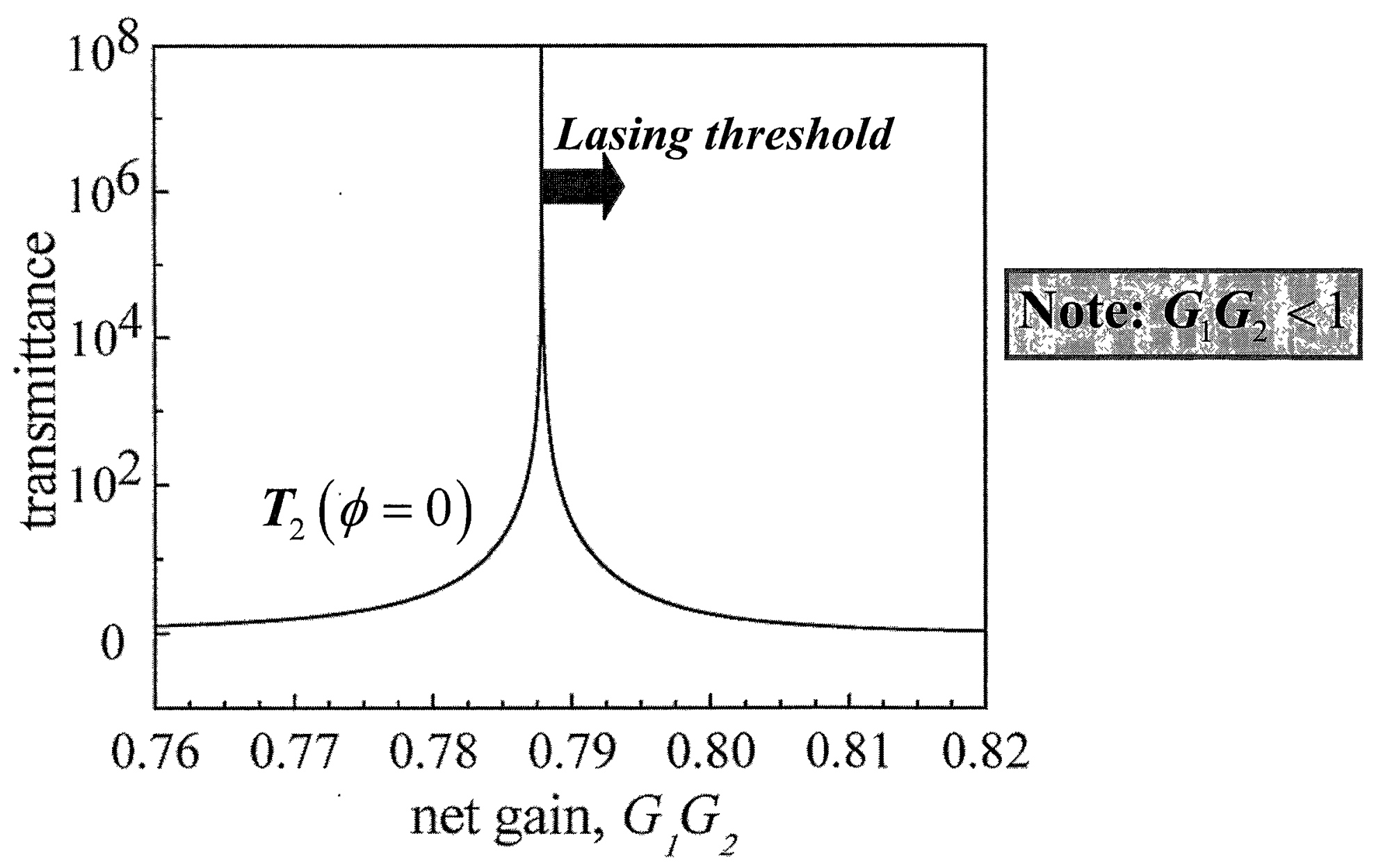




\section{Reduced Thresholds in Coupled Resonators}

- Threshold for coupled resonators $<$ threshold for single resonator

- Resonators must be properly phased for constructive interference

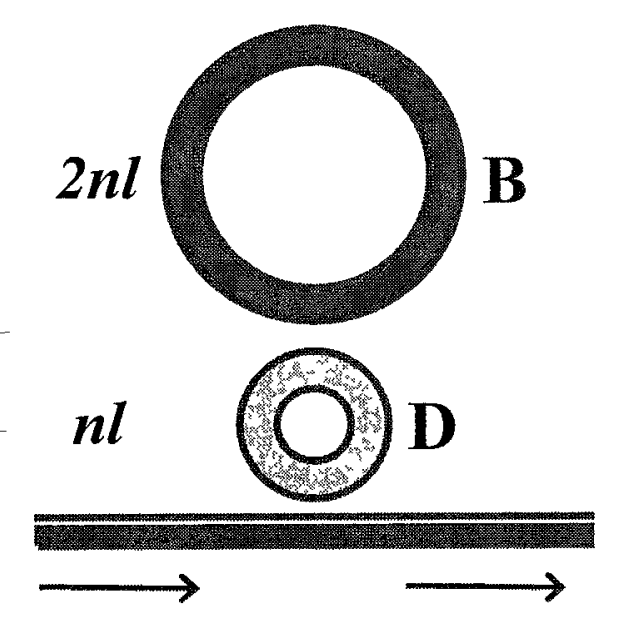

Upper resonator twice OPL of lower

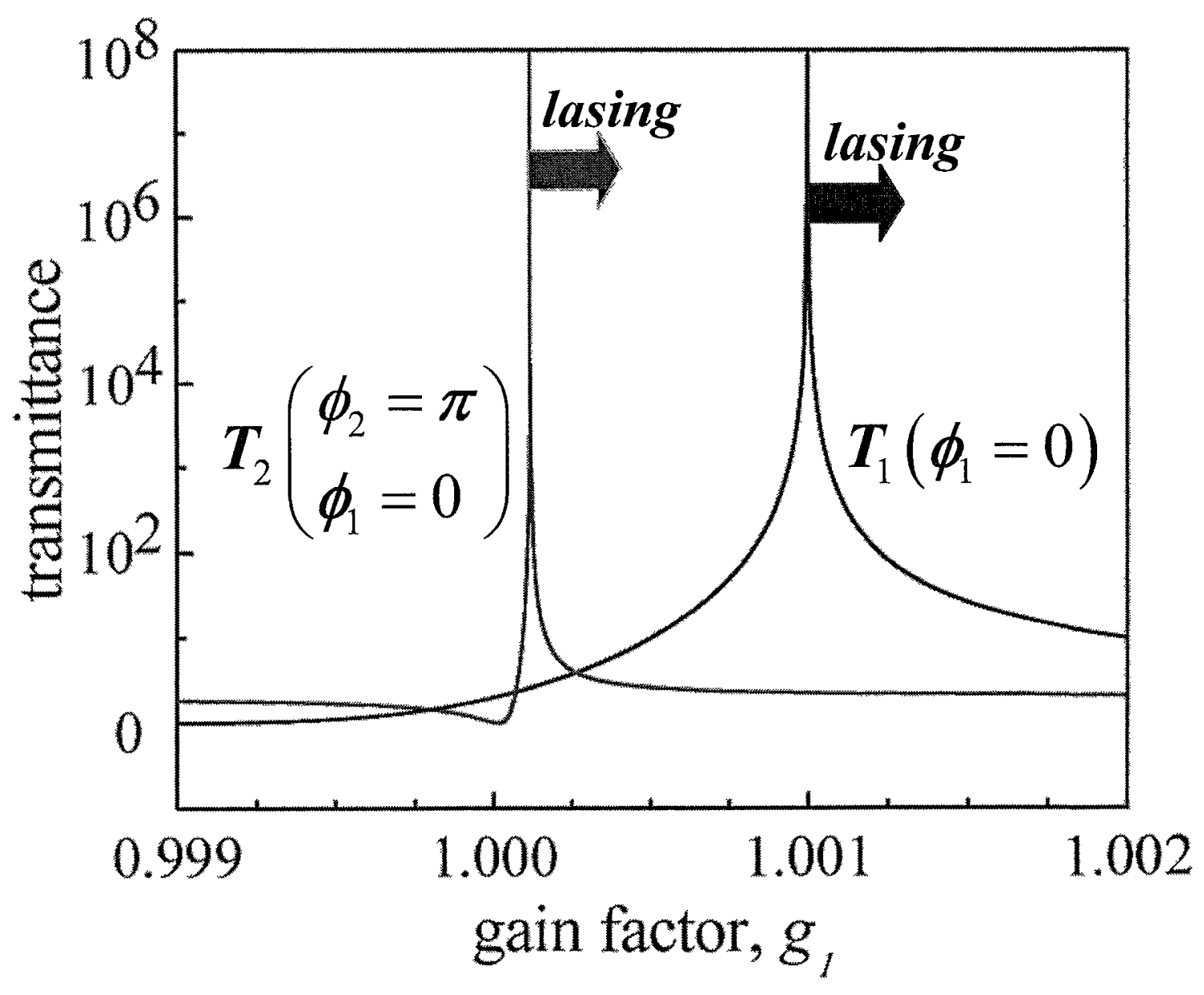




\section{- Beat Frequency and Relative Modulation}

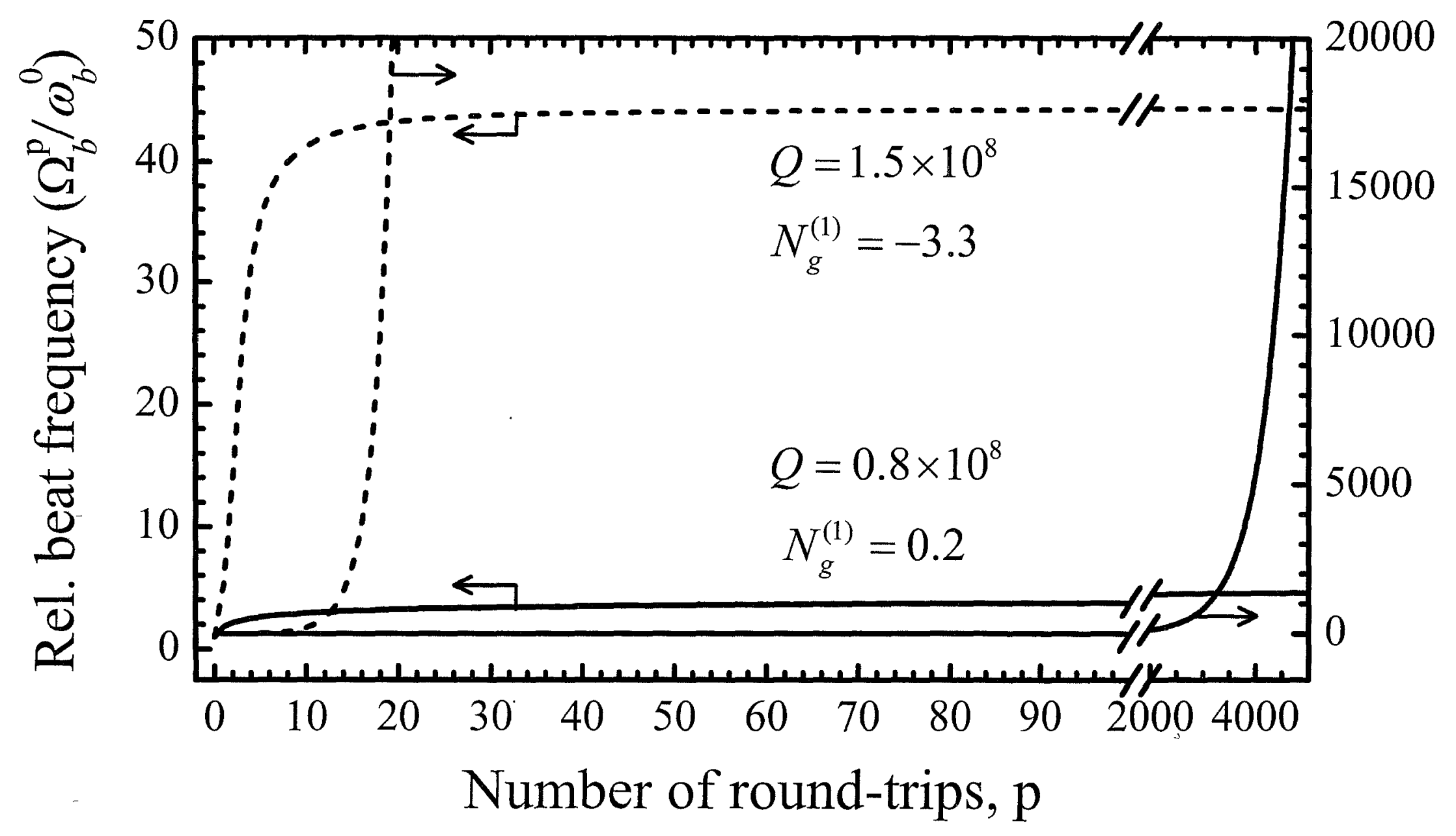

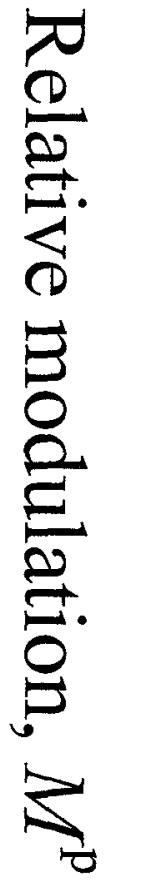




\section{Laser Gyro Enhancement}

$$
Q=0.8 \times 10^{8}
$$

$>$ Scale-Factor increased by $1 / N$

$>$ Elimination of Gyro Dead-band

> Single Resonators: under-coupled (Anomalous) or overcoupled (Normal), but no gain.

$>$ Coupled Resonators: CRIA or GAS but not CRIT.

$$
N_{g}^{(1)}=0.2
$$

Effective phase shift, $\Phi(\pi \mathrm{rad})$

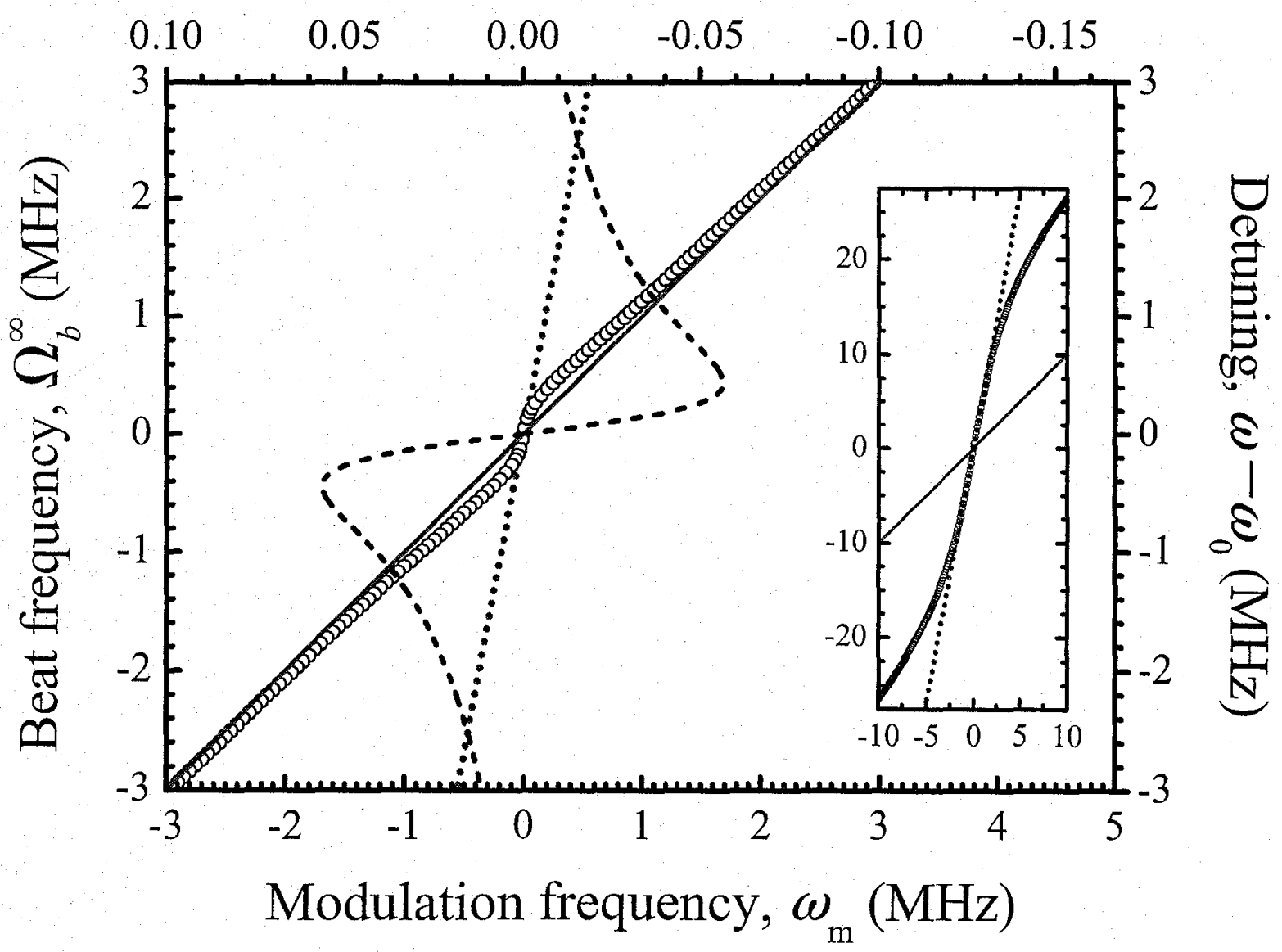




\section{- Summary and Conclusions}

$>$ Coupled resonators are analogous to multilevel atoms and are described approximately by the damped Rabi problem.

$>$ Photons can be shuffled from one resonator to another using coherent and adiabatic photon transfer techniques. Coupled resonators can store light.

$>$ Coherence phenomena such as EIT, EIA, GAS, and LWI are fundamental to systems of coherently coupled oscillators. They are not unique to atoms nor are they uniquely quantum phenomena.

$>$ The dispersion in optical micro-resonators has application to the improvement of laser gyroscopes. 


\section{Acknowledgements}

Collaborators:

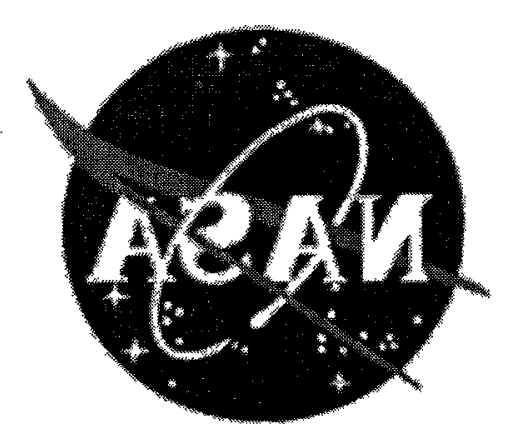

Support:

$>$ MSFC Institutional Research and Development Fund

$>$ United Negro College Fund Office of Special Programs

$>$ NASA Administrator's Fellowship Program

$>$ MSFC Education Programs Office 